\title{
HESSIAN EQUATIONS WITH ELEMENTARY SYMMETRIC FUNCTIONS
}

\author{
HONGJIE DONG
}

\begin{abstract}
We consider the Dirichlet problem for two types of degenerate elliptic Hessian equations. New results about solvability of the equations in the $C^{1,1}$ space are provided.
\end{abstract}

\section{INTRODUCTION}

This article is closely related to [1], [7 and [15]. A second-order partial differential equation is called Hessian equation if it is of the form

$$
F\left(u_{x x}\right)=f
$$

where $\left(u_{x x}\right)$ is the Hessian matrix of $u$ and $F(w)$ only depends on the eigenvalues of the symmetric matrix $w$.

Here we are concerned with the Dirichlet problem for two types of degenerate Hessian equations:

$$
\begin{gathered}
P_{m}\left(u_{x x}\right)=\sum_{k=0}^{m-1}\left(l_{k}^{+}\right)^{m-k}(x) P_{k}\left(u_{x x}\right), \\
P_{m}\left(u_{x x}\right)=g^{m-1},
\end{gathered}
$$

where $P_{k}\left(u_{x x}\right)$ is the $k$ th elementary symmetric polynomial of eigenvalues of the matrix $u_{x x}$, and $l_{k}, g$ are in $C^{1,1}$. The second equation is called $m$-Hessian equation, which becomes the Monge-Ampère equation when $m=d$. Equation (1.2) is non-degenerate if $g>0$ and is degenerate if $g$ can vanish at some points. Many authors have studied the Hessian equations and especially the Monge-Ampère equation. The solvability of non-degenerate equations is proved by establishing the $C^{2, \alpha}$ estimate of the solutions and using the method of continuity (see [1], 13] and [14]). For non-degenerate equations, we also refer the

Date: July 15, 2018.

1991 Mathematics Subject Classification. 35J60.

Key words and phrases. Degenerate Hessian equations; Dirichlet problem; Weak solutions. 
reader to the works [10, [1, 3] and [6]. It is well-known that there exists a unique admissible weak solution to degenerate problem (see [22]). To show the existence and uniqueness of solutions in $C^{1,1}$, it suffices to obtain a priori estimate of the second order derivatives of smooth solutions to the approximating non-degenerate equations (cf. Lemma 4.6).

For the degenerate case, a global upper bound for the second-order derivatives of admissible solutions to the Dirichlet problem of Hessian equations, and more general Bellman equations, was established by N.V. Krylov in a series of papers [15-18]. In [15] a few concrete equations similar to (1.1) and (1.2) like

$$
\begin{gathered}
P_{m}\left(u_{x x}\right)=\sum_{k=0}^{m-1}\left(l_{k}^{+}\right)^{m-k+1}(x) P_{k}\left(u_{x x}\right), \\
P_{m}\left(u_{x x}\right)=\left(l_{k}^{+}\right)^{m-k}(x) P_{k}\left(u_{x x}\right), \quad k<m,
\end{gathered}
$$

and, in particular, $P_{m}\left(u_{x x}\right)=\left(g_{+}\right)^{m}$ as $k=0$, are treated as the applications of the general theory.

Later, for the case of the Monge-Ampère equation

$$
P_{d}\left(u_{x x}\right)=\operatorname{det}\left(u_{x x}\right)=g^{d-1},
$$

the solvability in the $C^{1,1}$ space was proved in 7] by P. Guan, N.S. Trudinger and X-J. Wang with a different approach. The power $d-1$ of $g$ was also shown to be optimal by an example in [23. A modification of such example shows that $m-1$ is the lowest possible power of $g$ when there exist second derivatives estimates for solutions of the $\mathrm{m}$ Hessian equations (see also recent [11, where the authors did a very good survey of the literature on the Hessian equations). As pointed out in [7, the techniques there rest on the fact that the solution is convex in case $m=d$, which in general does not always hold true for $m$-Hessian equations. With $g^{m-1}$ on the right-hand side, the solvability of the general degenerate $m$-Hessian equations in the $C^{1,1}$ space is still unknown.

The purpose of this paper is to prove the solvability of the Dirichlet problems of type (1.1) with $C^{3,1}$ boundary data, and also the Dirichlet problems for the degenerate $m$-Hessian equations (1.2) with homogeneous boundary data. Our results improve the corresponding results in [15. Quite a few arguments in the paper are based on or follow the results in [15] and 17. The technique we use is to reduce the Hessian equations to the elliptic Bellman's equations and then apply the general theorems on the Bellman equations, which were introduced in [15]. 
Owing to an observation that a certain function is quasiconvex (Theorem 3.4) we are able to apply this technique to show the solvability of equation (1.2).

The article is organized as follows. Our two main theorems 2.5 and 2.6) are given in the following section. Theorem 2.5] is proved in Section 3. We prove some preliminary results and give the estimates of $u, u_{x}$ in Section 4 and 5. In Section 6. we use the maximum principle in a sub-domain and reduce the estimation of second order derivatives to the estimation of their values on the boundary. After that, the boundary second derivatives are estimated in Section 7 in a standard way combining with a Hopf type lemma (Lemma 5.3), and this completes the proof of Theorem 2.6.

To conclude the introduction, we explain some notation used in what follows: $\mathbb{R}^{d}$ is a $d$-dimensional Euclidean space with a fixed orthonormal basis. A typical point in $\mathbb{R}^{d}$ is denoted by $x=\left(x^{1}, x^{2}, \ldots, x^{d}\right)$. As usual the summation convention over repeated indices is enforced. For any $l=\left(l^{1}, l^{2}, \ldots, l^{d}\right) \in \mathbb{R}^{d}$ and any differentiable function $u$ on $\mathbb{R}^{d}$, we denote $D_{l} u=u_{x^{i}} l^{i}$ and $D_{l}^{2} u=u_{x^{i} x^{j}} l^{i} l^{j}$, etc.

Let $d \geq 2, m$ be positive integers, $2 \leq m \leq d$. We denote by $\mathbb{S}^{d}$ the set of all symmetric $d \times d$ matrices, $\mathbb{R}_{+}\left(\mathbb{R}_{+}^{0}\right)$ the set of all nonnegative (strictly positive) real numbers and $\mathcal{M}_{+}\left(\mathcal{M}_{+}^{0}\right)$ the set of all nonnegative (strictly positive) symmetric $d \times d$ matrices.

Various constants are denoted by $N$ and $\delta$ in general and the expression $N=N(\cdot)$ means that the given constant $N$ depends only on the contents of the parentheses.

\section{ACKNOWLEDGEMENT}

The author would like to express his sincere gratitude to his advisor, N.V. Krylov, for pointing out this problem and giving many useful suggestions and comments for improvements. The author is also grateful to the anonymous referee who gave very useful comments on the first submission of the article.

\section{The Setting And Main Results}

Define $P_{m}(\lambda)=P_{m, d}(\lambda)$ as the $m$ th elementary symmetric polynomial of the variables $\lambda=\left(\lambda^{1}, \lambda^{2}, \ldots, \lambda^{d}\right)$. For any symmetric $d \times d$ matrix $w$, define $\lambda(w)$ as a vector of eigenvalues of $w$ with arbitrary order and define $P_{m}(w)=P_{m}(\lambda(w))$. Let $C_{m}=C_{m, d}$ be the open connected component of the set $\left\{w \in \mathbb{S}^{d}: P_{m}(w)>0\right\}$ which contains the identity matrix $I$. 
Definition 2.1. Suppose $D:=\left\{x \in \mathbb{R}^{d} \mid \psi(x)>0\right\}$ is a smooth bounded domain with connected boundary. We say $D$ is $m-1$-convex if for a large number $K$ and any point on $\partial D$ we have

$$
P_{m-1, d-1}\left(\kappa^{1}, \ldots, \kappa^{d-1}\right) \geq 1 / K,
$$

where $\kappa^{1}, \ldots, \kappa^{d-1}$ are the principle curvatures of $\partial D$ at this point evaluated with respect to the interior normal to $\partial D$.

Definition 2.2. A function $u \in C^{2}(D)$ is called $m$-admissible if the Hessian matrix $\left(u_{x x}\right)$ is in $\bar{C}_{m}$ for any $x \in D$. A function $u \in C^{1,1}(D)$ is called $m$-admissible if the Hessian matrix of the second order Sobolev derivatives $\left(u_{x x}\right)$ is in $\bar{C}_{m}$ for almost any $x \in D$.

In this article, we always assume that $D$ is an $m-1$-convex bounded domain of class $C^{3,1}$ with connected boundary. We are concerned with the following two Dirichlet problems for the elliptic Hessian equations,

$$
\begin{gathered}
P_{m}\left(u_{x x}\right)=\sum_{k=0}^{m-1}\left(l_{k}^{+}\right)^{m-k}(x) P_{k}\left(u_{x x}\right) \text { (a.e.) in } D, \\
u=\phi \text { on } \partial D
\end{gathered}
$$

where $l_{0}, \ldots, l_{m-1}$ are bounded real valued functions in $\mathbb{R}^{d}$ and $\phi \in$ $C^{3,1}\left(\mathbb{R}^{d}\right)$, and the $m$-Hessian equation,

$$
\begin{aligned}
P_{m}\left(u_{x x}\right) & =g^{m-1} & & \text { in } D, \\
u & =\phi & & \text { on } \partial D,
\end{aligned}
$$

where $2 \leq m \leq d, g \in C^{1}(\bar{D})$ is nonnegative, $\phi \in C^{3,1}(\bar{D})$.

Assumption 2.3. For some sufficiently large number $K$ and any $k=$ $0,1, \cdots, m-1$ the functions $l_{k}(x)+K|x|^{2}$ and $g(x)+K|x|^{2}$ are convex on $\bar{D}$, and

$$
\begin{gathered}
\operatorname{diam}\{D\}+\|g\|_{C^{1}(\bar{D})}+1 / \sup _{\bar{D}}|g|+\|\phi\|_{C^{3,1}(\bar{D})}+\|\psi\|_{C^{3,1}}+\left\|l_{k}\right\|_{C^{0}} \leq K \\
\left|\psi_{x}\right| \geq 1 / K \quad \text { on } \partial D \\
|\nabla g(x)|^{2} \leq K g(x) \quad \text { in } D
\end{gathered}
$$

Remark 2.4. Typically, inequality (2.6) holds if $g \in C^{1,1}\left(\mathbb{R}^{d}\right)$ is nonnegative and $\|g\|_{C^{1,1}} \leq K / 2$. We note here that (2.6) is also needed in [7] as well, for example, in the proof of Lemma 2.1 there. Because of that the result of [7] does not completely cover the result in [15] about $\operatorname{det}\left(u_{x x}\right)=\left(g_{+}\right)^{d}$ since $\left(g_{+}\right)^{d /(d-1)}$ may not satisfy (2.6) .

Here come our two main results. 
Theorem 2.5. Under the above assumptions, equation (2.2)-(2.3) has a unique solution $u \in C^{1,1}(\bar{D})$ characterized by the additional property that

$$
\begin{gathered}
P_{m}\left(u_{x^{i} x^{j}}+t \delta_{i j}\right)>0, \\
P_{m}\left(u_{x^{i} x^{j}}+t \delta_{i j}\right)>\sum_{k=0}^{m-1}\left(l_{k}^{+}\right)^{m-k}(x) P_{k}\left(u_{x^{i} x^{j}}+t \delta_{i j}\right) \text { (a.e.) in } D,
\end{gathered}
$$

for any $t>0$. Moreover, if $\sum_{k} l_{k}^{+}>0$ in $\bar{D}$, then $u \in C^{2, \alpha}(\bar{D})$ for an $\alpha \in(0,1)$.

Theorem 2.6. Under the above assumptions, there exists a unique $m$-admissible solution $u \in C^{1,1}(\bar{D})$ of the Dirichlet problem (2.4) $-(2.5)$ with homogeneous boundary condition, i.e. $\phi \equiv 0$. Moreover, the solution $u$ satisfies (2.4) almost everywhere in $D$ and admits an estimate

$$
\|u\|_{C^{1,1}(\bar{D})} \leq N(D, d, K) .
$$

Assumption 2.3 and in addition we assume more $g$ near $\partial D$, that is for some large $K_{1}$,

\section{Proof of Theorem 2.5]}

Firstly, let's restate some results, which can be found, for instance, in [15], as the following two lemmas.

Lemma 3.1. The set $C_{m}$ defined above is an open convex cone in $\mathbb{S}^{d}$ with vertex at the origin containing $\mathcal{M}_{+}^{0}$, and for $k=0,1, \ldots, m-1$ we have $C_{m} \subset C_{k}$. Furthermore, the functions $\left(P_{k} / P_{k-1}\right)(w), k=$ $2,3, \ldots, m$, are concave in $C_{m}$.

Lemma 3.2. If a function $F(w)$ is convex and homogeneous of degree $-\alpha$ in a cone $C \subset \mathbb{S}^{d}$, then the function $l^{\alpha+1} F(w)$ is a convex function of $(w, l)$ in $C \times \mathbb{R}_{+}$.

The following lemma can be easily proved by direct calculation of the Hessian matrix.

Lemma 3.3. For any positive number $n$, the function

$$
\left((x+y)^{n}-x^{n}\right)^{-1}
$$

is a convex function in the open cone $\mathbb{R}_{+}^{0} \times \mathbb{R}_{+}^{0}$.

As a corollary of Lemma 3.2 and 3.3 , the function

$$
H(x, y, l):=l^{n+1} /\left((x+y)^{n}-x^{n}\right)
$$

is convex in $\mathbb{R}_{+}^{0} \times \mathbb{R}_{+}^{0} \times \mathbb{R}_{+}$.

The proof of Theorem 2.5 relies on the following observation. 
Theorem 3.4. We denote

$$
G(w, l)=\sum_{k=0}^{m-1}\left(l_{k}^{m-k}\right)\left(P_{k} / P_{m}\right)(w)
$$

where $l=\left(l_{0}, l_{1}, \ldots l_{m-1}\right)$. Then for any $(w, l),(\tilde{w}, \tilde{l}) \in C_{m} \times \mathbb{R}_{+}^{m}$, we have

$$
G\left(\frac{w+\tilde{w}}{2}, \frac{l+\tilde{l}}{2}\right) \leq \max (G(w, l), G(\tilde{w}, \tilde{l})) .
$$

That is, $G(w, l)$ is quasiconvex in $C_{m} \times \mathbb{R}_{+}^{m}$.

Proof. Suppose for some $c \geq 0$, the following two inequalities hold

$$
\sum_{k=0}^{m-1} l_{k}^{m-k} P_{k}(w) \leq c P_{m}(w), \sum_{k=0}^{m-1} \tilde{l}_{k}^{m-k} P_{k}(\tilde{w}) \leq c P_{m}(\tilde{w}) .
$$

Due to the homogeneity, to prove the theorem it suffices to prove the inequality

$$
\sum_{k=0}^{m-1}\left(l_{k}+\tilde{l}_{k}\right)^{m-k} P_{k}(w+\tilde{w}) \leq c P_{m}(w+\tilde{w}) .
$$

For $k=0, \ldots, m-1$, let $\alpha_{k}, \tilde{\alpha}_{k}$ be the nonnegative numbers such that

$$
\begin{aligned}
& \left(l_{k}+\alpha_{k}\right)^{m-k} P_{k}(w)=\sum_{j=0}^{k} l_{j}^{m-j} P_{j}(w), \\
& \left(\tilde{l}_{k}+\tilde{\alpha}_{k}\right)^{m-k} P_{k}(\tilde{w})=\sum_{j=0}^{k} \tilde{l}_{j}^{m-j} P_{j}(\tilde{w}) .
\end{aligned}
$$

Then,

$$
\left(l_{m-1}+\alpha_{m-1}\right) P_{m-1}(w) \leq c P_{m}(w),\left(\tilde{l}_{m-1}+\tilde{\alpha}_{m-1}\right) P_{m-1}(\tilde{w}) \leq c P_{m}(\tilde{w}) .
$$

Owing to the concavity of $\left(P_{m} / P_{m-1}\right)(w)$, we get

$$
\begin{gathered}
c\left(P_{m} / P_{m-1}\right)(w+\tilde{w}) \geq c\left(P_{m} / P_{m-1}\right)(w)+c\left(P_{m} / P_{m-1}\right)(\tilde{w}) \\
\geq l_{m-1}+\alpha_{m-1}+\tilde{l}_{m-1}+\tilde{\alpha}_{m-1}, \\
c P_{m}(w+\tilde{w}) \geq\left(l_{m-1}+\alpha_{m-1}+\tilde{l}_{m-1}+\tilde{\alpha}_{m-1}\right) P_{m-1}(w+\tilde{w}) .
\end{gathered}
$$

By using (3.2) and (3.3), for $k=1, \ldots, m-1$ we have

$$
\begin{aligned}
& \left(\left(l_{k}+\alpha_{k}\right)^{m-k}-l_{k}^{m-k}\right) P_{k}(w)=\left(l_{k-1}+\alpha_{k-1}\right)^{m-k+1} P_{k-1}(w), \\
& \left(\left(\tilde{l}_{k}+\tilde{\alpha}_{k}\right)^{m-k}-\tilde{l}_{k}^{m-k}\right) P_{k}(\tilde{w})=\left(\tilde{l}_{k-1}+\tilde{\alpha}_{k-1}\right)^{m-k+1} P_{k-1}(\tilde{w}) .
\end{aligned}
$$


For any $\varepsilon>0$, the equalities above imply the following

$$
\begin{aligned}
& \left(\left(l_{k}+\alpha_{k}+2 \varepsilon\right)^{m-k}-\left(l_{k}+\varepsilon\right)^{m-k}\right) P_{k}(w) \geq\left(l_{k-1}+\alpha_{k-1}\right)^{m-k+1} P_{k-1}(w), \\
& \left(\left(\tilde{l}_{k}+\tilde{\alpha}_{k}+2 \varepsilon\right)^{m-k}-\left(\tilde{l}_{k}+\varepsilon\right)^{m-k}\right) P_{k}(\tilde{w}) \geq\left(\tilde{l}_{k-1}+\tilde{\alpha}_{k-1}\right)^{m-k+1} P_{k-1}(\tilde{w}) .
\end{aligned}
$$

Again, owing to the concavity of $\left(P_{k} / P_{k-1}\right)(w)$ in $C_{m}$ and the corollary of Lemma 3.3, we obtain

$$
\begin{aligned}
& \left(P_{k} / P_{k-1}\right)(w+\tilde{w}) \geq\left(P_{k} / P_{k-1}\right)(w)+\left(P_{k} / P_{k-1}\right)(\tilde{w}) \\
& \geq \frac{\left(l_{k-1}+\alpha_{k-1}\right)^{m-k+1}}{\left(l_{k}+\alpha_{k}+2 \varepsilon\right)^{m-k}-\left(l_{k}+\varepsilon\right)^{m-k}}+\frac{\left(\tilde{l}_{k-1}+\tilde{\alpha}_{k-1}\right)^{m-k+1}}{\left(\tilde{l}_{k}+\tilde{\alpha}_{k}+2 \varepsilon\right)^{m-k}-\left(\tilde{l}_{k}+\varepsilon\right)^{m-k}} \\
& \geq \frac{\left(l_{k-1}+\alpha_{k-1}+\tilde{l}_{k-1}+\tilde{\alpha}_{k-1}\right)^{m-k+1}}{\left(l_{k}+\alpha_{k}+\tilde{l}_{k}+\tilde{\alpha}_{k}+4 \varepsilon\right)^{m-k}-\left(l_{k}+\tilde{l}_{k}+2 \varepsilon\right)^{m-k}} .
\end{aligned}
$$

As a consequence,

$$
\begin{gathered}
\left(\left(l_{k}+\alpha_{k}+\tilde{l}_{k}+\tilde{\alpha}_{k}+4 \varepsilon\right)^{m-k}-\left(l_{k}+\tilde{l}_{k}+2 \varepsilon\right)^{m-k}\right) P_{k}(w+\tilde{w}) \\
\geq\left(l_{k-1}+\alpha_{k-1}+\tilde{l}_{k-1}+\tilde{\alpha}_{k-1}\right)^{m-k+1} P_{k-1}(w+\tilde{w}) .
\end{gathered}
$$

Letting $\varepsilon \downarrow 0$ and taking the limit yield

$$
\begin{aligned}
& \left(\left(l_{k}+\alpha_{k}+\tilde{l}_{k}+\tilde{\alpha}_{k}\right)^{m-k}-\left(l_{k}+\tilde{l}_{k}\right)^{m-k}\right) P_{k}(w+\tilde{w}) \\
& \geq\left(l_{k-1}+\alpha_{k-1}+\tilde{l}_{k-1}+\tilde{\alpha}_{k-1}\right)^{m-k+1} P_{k-1}(w+\tilde{w}) .
\end{aligned}
$$

Inequality (3.1) follows if we add (3.4) and (3.5) together, and the theorem is proved.

Finally, by relying on Theorem 3.4 at one point, we can essentially reproduce Krylov's approach in [15] with very few modifications (cf. Remark 5.14, 5.16 of [15]). The idea is to reduce the equation to a Bellman's equation and apply a general existence and uniqueness result on degenerate elliptic Bellman's equations proved in 18 by a probabilistic argument, or by an analytic approach in [16] and [17. In detail, due to Theorem 3.4 we can easily get that

$$
\Theta(l):=\left\{w \in C_{m}: G(w, l)<1\right\}
$$

is convex in $l$ in the sense that for any $w_{i} \in \Theta\left(l_{i}\right), i=1,2$ we have

$$
\frac{w_{1}+w_{2}}{2} \in \Theta\left(\frac{l_{1}+l_{2}}{2}\right) .
$$

Then after one reduces the equation to a Bellman's equation, the free term is semi-concave so that the regularity theory for Bellman's equations is applicable. Theorem 2.5 improves the corresponding result in [15] also from the point of view of the following remark. 
Remark 3.5. The same conclusion as in Theorem 2.5 holds true if we replace (2.2) by

$$
P_{m}\left(u_{x x}\right)=\sum_{k=0}^{m-1} f_{k}\left(l_{k}^{+}(x)\right) P_{k}\left(u_{x x}\right) \text { (a.e.) in } D,
$$

for any $s \geq 0$, where $f_{k}: \mathbb{R}_{+} \rightarrow \mathbb{R}_{+}$are continuous functions and $f_{k}^{1 /(m-k)}$ are convex for $k=0,1, \cdots, m-1$.

Indeed, if we denote

$$
G_{1}(w, l)=\sum_{k=0}^{m-1} f_{k}\left(l_{k}\right)\left(P_{k} / P_{m}\right)(w)
$$

then due to Theorem 3.4 and the convexity of $f_{k}^{1 /(m-k)}$ in $\mathbb{R}_{+}$, for any $(w, l),(\tilde{w}, \tilde{l}) \in C_{m} \times \mathbb{R}_{+}^{m}$ we have

$$
\begin{gathered}
\max \left(G_{1}(w, l), G_{1}(\tilde{w}, \tilde{l})\right) \\
=\max \left\{\sum_{k} f_{k}\left(l_{k}\right)\left(P_{k} / P_{m}\right)(w), \sum_{k} f_{k}\left(\tilde{l}_{k}\right)\left(P_{k} / P_{m}\right)(\tilde{w})\right\} \\
\geq \sum_{k}\left[\frac{1}{2}\left(f_{k}^{1 /(m-k)}\left(l_{k}\right)+f_{k}^{1 /(m-k)}\left(\tilde{l}_{k}\right)\right)\right]^{m-k}\left(P_{k} / P_{m}\right)\left(\frac{w+\tilde{w}}{2}\right) \\
\geq \sum_{k} f_{k}\left(\frac{l_{k}+\tilde{l}_{k}}{2}\right)\left(P_{k} / P_{m}\right)\left(\frac{w+\tilde{w}}{2}\right)=G_{1}\left(\frac{w+\tilde{w}}{2}, \frac{l+\tilde{l}}{2}\right),
\end{gathered}
$$

i.e. $G_{1}(w, l)$ is also quasiconvex in $C_{m} \times \mathbb{R}_{+}^{m}$. Thus our assertion follows.

\section{Some Preliminary Results}

In what follows, we consider the following Dirichlet problem for $2 \leq$ $m \leq d$ :

$$
\begin{aligned}
& P_{m}\left(u_{x x}\right)=g^{m-1} \quad \text { in } D \text {, } \\
& u=0 \quad \text { on } \partial D \text {, }
\end{aligned}
$$

where $g \in C^{1,1}(\bar{D})$ is nonnegative and $P_{m}$ is defined in Section 2. We focus on the solution $u$ such that $\left(u_{x x}\right) \in \bar{C}_{m}$ for any $x \in D$.

Lemma 4.1. For any matrix $\left(v_{i j}\right)$ in $C_{m}$, the $d$ by $d$ matrix $K(v):=$ $\left(P_{m, v_{i j}}(v)\right)$ is positive definite.

Proof. Let $\eta$ be a nonzero vector in $\mathbb{R}^{d}$. We have

$$
\eta^{T} K(v) \eta=\operatorname{Tr}\left(K(v) \eta \eta^{T}\right)=\left.\frac{d}{d t} P_{m}\left(v+t \eta \eta^{T}\right)\right|_{t=0} .
$$


Since the rank of $\eta \eta^{T}$ is one, $P_{m}\left(v+t \eta \eta^{T}\right)$ is linear in $t$. Thus,

$$
P_{m}\left(v+t \eta \eta^{T}\right)=P_{m}(v)+t \eta^{T} K(v) \eta
$$

Also note that $\eta \eta^{T} \in \bar{C}_{d} \subset \bar{C}_{m}$, for any $t \geq 0$ we have

$$
v+t \eta \eta^{T} \in \bar{C}_{m}, \quad P_{m}\left(v+t \eta \eta^{T}\right) \geq 0 .
$$

Therefore, $\eta^{T} K(v) \eta \geq 0$ is nonnegative. If $\eta^{T} K(v) \eta=0$, for any $t>0$ it holds that

$$
P_{m}\left(v-t \eta \eta^{T}\right)=P_{m}(v)>0,
$$

which implies $v-t \eta \eta^{T} \in C_{m}$ and thus $v / t-\eta \eta^{T} \in C_{m}$. Letting $t \rightarrow+\infty$ yields $-\eta \eta^{T} \in \bar{C}_{m} \in \bar{C}_{1}$. But this is impossible because

$$
P_{1}\left(-\eta \eta^{T}\right)=\operatorname{Tr}\left(-\eta \eta^{T}\right)=-|\eta|^{2}<0 .
$$

Hence it holds that $\eta^{T} K(v) \eta>0$, and the lemma is proved.

The following corollary is an immediate consequence of Lemmas 3.1 and 4.1 .

Corollary 4.2. For any $d \times d$ matrix $\left(v_{i j}\right)$ in $C_{m}$, all the $(d-1) \times(d-1)$ submatricies obtained by deleting the kth row and kth column of $\left(v_{i j}\right)$ $(k=1, \cdots, d)$ are in $C_{m-1, d-1}$.

Lemma 4.3. (i) For any matrices $\left(v_{i j}\right)$ and $\left(w_{i j}\right)$ in $C_{m}$, we have

$$
\operatorname{Tr}(K(v) w)=P_{m, v_{i j}}(v) w_{i j}>0 .
$$

(ii) Moreover, for any orthogonal matrix $Q$, it holds that

$$
K\left(Q v Q^{T}\right)=Q K(v) Q^{T} .
$$

Proof. The key idea of the proof of part (i) is to use the properties of hyperbolic polynomials. We treat $P_{m}(v)$ as a homogenous polynomial of $d(d+1) / 2$ variables

$$
v_{11}, v_{12}, \ldots, v_{1 d}, v_{22}, v_{23}, \ldots, v_{2 d}, v_{33}, \ldots, v_{3 d}, \ldots, v_{d d} .
$$

It's known that for any $\mu \in \mathbb{R}^{d}$, all roots of the polynomial $P_{m}\left(\mu+t \lambda_{0}\right)$ are real, where $\lambda_{0}=(1,1, \ldots, 1)$ (cf. Corollary 6.5 of [15). Therefore, by the very definition of $P_{m}$ in $\mathbb{S}^{d}$, for any $v \in \mathbb{S}^{d}$, all roots of the polynomial $P_{m}\left(v+t I_{d}\right)$ are real. Owing to Lemma 4.16 and Theorem 6.4 of [15], for any $v, w$ in $C_{m}$ we have

$$
P_{m, v_{i j}}(v) w_{i j}=\left.\frac{d}{d t} P_{m}(v+t w)\right|_{t=0}>0 .
$$

This proves the first part of the lemma. 
To prove the second part, first notice that $P_{m}$ and $C_{m}$ are both invariant under orthogonal transformations. Suppose $w$ is a symmetric matrix. We have

$$
\begin{gathered}
\operatorname{Tr}\left(K\left(Q v Q^{T}\right) w\right)=\left.\frac{d}{d t} P_{m}\left(Q v Q^{T}+t w\right)\right|_{t=0}=\left.\frac{d}{d t} P_{m}\left(v+t Q^{T} w Q\right)\right|_{t=0} \\
=\operatorname{Tr}\left(K(v) Q^{T} w Q\right)=\operatorname{Tr}\left(Q K(v) Q^{T} w\right) .
\end{gathered}
$$

Since $w$ is an arbitrary symmetric matrix, the conclusion of (ii) follows immediately.

Remark 4.4. By using the same method as in the proof of 4.3 (i), owing to Lemma 4.16 and Theorem 6.4 of [15], one can prove that for any $\left(v_{i j}\right),\left(w_{i j}\right)$ in $C_{m}$ and $t_{0}, s_{0} \geq 0$ it holds that

$$
\left.\frac{\partial^{2}}{\partial t \partial s} P_{m}(s v+t w)\right|_{(s, t)=\left(s_{0}, t_{0}\right)} \geq 0 .
$$

And this implies

$$
P_{m}(v+w) \geq P_{m}(v)+P_{m}(w) .
$$

Lemma 4.5. Let $v \in \bar{C}_{m}$ and $c$ be a nonnegative constant. Then $P_{m}(v)=c$ if and only if

$$
\inf _{w \in C_{m}}\left\{a^{i j}(w) v^{i j}-m(d-m+1)^{-1} P_{m}^{1-1 / m}(w) P_{m-1}^{-1}(w) c^{1 / m}\right\}=0,
$$

where

$$
a^{i j}(w)=P_{m, w^{i j}}(w) / \operatorname{Tr}(K(w))
$$

Proof. First we suppose $P_{m}(v)=c$. Recall that $P_{m}^{1 / m}(v)$ is concave in $C_{m}$ (see, for instance, Theorem 6.4 of [15]). We get

$$
\begin{gathered}
c^{1 / m}=P_{m}^{1 / m}(v) \\
=\inf _{w \in C_{m}}\left\{m^{-1} P_{m, w^{i j}}(w) P_{m}^{1 / m-1}(w)\left(v^{i j}-w^{i j}\right)+P_{m}^{1 / m}(w)\right\} \\
=\inf _{w \in C_{m}}\left\{m^{-1} P_{m, w^{i j}}(w) P_{m}^{1 / m-1}(w) v^{i j}(x)\right\} .
\end{gathered}
$$

The last equality is because $P_{m}$ is a homogeneous polynomial of degree $m$. In case $c>0$, because the infimum above is attained when $\omega=v$ and also because $\operatorname{Tr}(K(v))>0$, equality (4.5) follows immediately. If $c=0$, then one has $v \in \partial C_{m}$. Note that

$$
\operatorname{Tr}(K(v))=(d-m+1) P_{m-1}(v)
$$

and $v+\varepsilon I \in C_{m}$. For any real number $\varepsilon>0$, we have

$$
\begin{aligned}
& a^{i j}(v+\varepsilon I) v^{i j}=P_{m, v^{i j}}(v+\varepsilon I) v^{i j} / \operatorname{Tr}(K(v+\varepsilon I)) \\
& =P_{m, v^{i j}}(v+\varepsilon I)\left(v^{i j}+\varepsilon \delta_{i j}-\varepsilon \delta_{i j}\right) / \operatorname{Tr}(K(v+\varepsilon I))
\end{aligned}
$$




$$
=m(d-m+1)^{-1}\left(P_{m} / P_{m-1}\right)(v+\varepsilon I)-\varepsilon .
$$

Thanks to Lemma 4.16 (ii) of [15], we get

$$
\lim _{\varepsilon \downarrow 0} a^{i j}(v+\varepsilon I) v^{i j}=0,
$$

and (4.5) follows.

On the other hand, assume that (4.5) holds true. Observe that

$$
P_{m}(v+t I) \rightarrow+\infty \quad \text { as } t \rightarrow+\infty
$$

and there exists $t_{0} \leq 0$ such that $v+t_{0} I \in \partial C_{m}$ and $P_{m}\left(v+t_{0} I\right)=0$. By continuity one can find a real number $t_{1} \geq t_{0}$ such that $v+t_{1} I \in \bar{C}_{m}$ and $P_{m}\left(v+t_{1} I\right)=c$. Due to the first part of the proof, it holds that

$$
\begin{gathered}
\inf _{w \in C_{m}}\left\{\operatorname{Tr}\left(a(w)\left(v+t_{1} I\right)\right)-m(d-m+1)^{-1} P_{m}^{1-1 / m}(w) P_{m-1}^{-1}(w) c^{1 / m}\right\}=0, \\
\inf _{w \in C_{m}}\left\{\operatorname{Tr}(a(w) v)-m(d-m+1)^{-1} P_{m}^{1-1 / m}(w) P_{m-1}^{-1}(w) c^{1 / m}\right\}=-t_{1} .
\end{gathered}
$$

Therefore, from (4.5) we obtain $t_{1}=0$, and the lemma is proved.

Because of Lemma 4.5, equation (4.1) is equivalent to

$$
\inf _{w \in C_{m}}\left\{L^{w} u(x)+f(w, x)\right\}=0,
$$

where

$$
\begin{gathered}
L^{w} u(x)=a^{i j}(w) u_{x_{i} x_{j}}(x), \\
f(w, x)=-m(d-m+1)^{-1} P_{m}^{1-1 / m}(w) P_{m-1}^{-1}(w) g^{1-1 / m}(x) .
\end{gathered}
$$

Owing to Theorem 1.1 of [22, (4.1)-(4.2) has a unique admissible weak solution $u \in C^{0}(D)$ in the sense that for a sequence of $m$ admissible functions $u_{k} \in C^{2}(D)$ we have

$$
u_{k} \rightarrow u \text { in } C^{0}(D), \quad P_{m}\left(u_{k, x x}\right) \rightarrow g^{m-1} \text { in } L_{\mathrm{loc}}^{1}(D) .
$$

The following lemma will be proved in Section 8

Lemma 4.6. Under Assumption 2.3, if we can establish a priori $C^{2}$ estimate of solutions to non-degenerate problems with $C^{2}(\bar{D}) \in g>0$, which does not depend on the infimum of $g$ in $D$, then

(i) the admissible weak solution $u$ of (4.1)-(4.2) is in $C^{1,1}(\bar{D})$.

(ii) Moreover, u satisfies (4.1) almost everywhere in D.

Due to Lemma 4.6. from now on, we always assume that $g$ is positive on $\bar{D}$ and belongs to $C^{2}(\bar{D})$. In this case, it's known that $u \in C^{4}(D) \cap$ $C^{2}(\bar{D})$. Because the infimum in (4.8) is attained when $\omega=u_{x x}$, it is easy to see that (4.8) is equivalent to a uniformly elliptic Bellman equation

$$
\inf _{w \in C_{m}^{*}}\left\{L^{w} u(x)+f(w, x)\right\}=0,
$$


where

$$
C_{m}^{*}=\left\{w \in C_{m} \mid P_{m}(w) \geq \inf _{D} g^{m-1}, w \leq N\left(\|u\|_{C^{2}(\bar{D})}\right) I\right\}
$$

Let $\xi$ be a unit vector in $\mathbb{R}^{d}$. Denote $\lambda^{1}(x), \ldots, \lambda^{d}(x)$ to be the eigenvalues of the Hessian matrix $u_{x x}(x)$. Due to Lemma 3.1. we have

$$
\lambda^{1}+\lambda^{2}+\ldots+\lambda^{d}=\operatorname{Tr}\left(u_{x x}\right)=\Delta u=P_{1}\left(u_{x x}\right)>0 .
$$

We can get more than (4.10). Define $\Lambda_{2}$ as the open connected component of the set $\left\{\lambda \in \mathbb{R}^{d}: P_{2}(\lambda)>0\right\}$ which contains the vector $(1,1, \ldots, 1)$. Since $\left(u_{x x}\right) \in C_{m} \subset C_{2}$, we have $\lambda:=\left(\lambda^{1}, \lambda^{2}, \ldots, \lambda^{d}\right) \in \Lambda_{2}$. Obviously, $\lambda_{\varepsilon}:=(1, \varepsilon, \varepsilon, \ldots, \varepsilon)$ is also in $\Lambda_{2}$ for any $\varepsilon>0$. Thus by Theorem 6.4(i) in [15] with $\lambda_{\varepsilon}$ and $P_{2}$ in place of $\lambda_{1}$ and $Q_{m}$ respectively, we get

$$
0<\frac{\partial}{\partial t} P_{2}\left(\lambda+t \lambda_{\varepsilon}\right)=\lambda^{2}+\lambda^{3}+\ldots+\lambda^{d}+O(\varepsilon) .
$$

By letting $\varepsilon \downarrow 0$ and taking the limit, we get

$$
\lambda^{2}+\lambda^{3}+\ldots+\lambda^{d}=\operatorname{Tr}\left(u_{x x}\right)-\lambda^{1} \geq 0 .
$$

Of course, (4.11) remains true if we replace 1 by any $i=2,3, \ldots, d$.

If $\max _{i} \lambda^{i}(x)<1$, due to (4.10), we immediately get an estimate of $\lambda^{i}(x), i=1,2, \ldots, d$. And this yields the estimate of $\left(u_{x x}\right)$. Therefore, in the sequel we only consider the region

$$
D^{\prime}:=\left\{x \in D \mid \max _{i} \lambda^{i}(x) \geq 1\right\} .
$$

Due to (4.11), in $D^{\prime}$ we have $P_{1}\left(u_{x x}\right) \geq 1$.

Lemma 4.7. (i) For any $x \in D^{\prime}$, we have

$$
g^{m-2}(x) \leq \operatorname{Tr}\left(K\left(u_{x x}\right)\right) /(d-m+1) .
$$

(ii) For any $x \in D$, we have

$$
g^{m-2}(x) \leq N g^{m-3 / 2}(x) \leq N \operatorname{Tr}\left(K\left(u_{x x}\right)\right) /(d-m+1),
$$

where $N$ depends only on $K$.

Proof. Note that $\left(P_{k}(w) /\left(\begin{array}{l}d \\ k\end{array}\right)\right)$ is a log-concave function of $k$ for $k=$ $0,1, \ldots, m$ (cf., for instance, Corollary $6.5[15]$ ). So in $D^{\prime}$ we have

$$
\begin{aligned}
g^{m-2}(x) & =P_{m}^{(m-2) /(m-1)}\left(u_{x x}\right) \leq P_{m}^{(m-2) /(m-1)}\left(u_{x x}\right) P_{1}^{1 /(m-1)}\left(u_{x x}\right) \\
& \leq N P_{m-1}\left(u_{x x}\right)=N \operatorname{Tr}\left(K\left(u_{x x}\right)\right) /(d-m+1) .
\end{aligned}
$$

Also for any matrix $w \in C_{m}$,

$$
P_{m}^{1-1 / m}(w)=P_{0}^{1 / m}(w) P_{m}^{(m-1) / m}(w) \leq N P_{m-1}(w) .
$$


Thus in $D$, we have

$$
\begin{gathered}
g^{m-3 / 2}(x) \leq N g^{(m-1)^{2} / m}(x)=N P_{m}^{(m-1) / m}\left(u_{x x}(x)\right) \\
\leq N P_{m-1}\left(u_{x x}(x)\right)=N \operatorname{Tr}\left(K\left(u_{x x}\right)\right) /(d-m+1) .
\end{gathered}
$$

The lemma is proved

Due to Assumption 2.3 and (4.14), for any $w \in C_{m}$ we have

$$
\|f(w, \cdot)\|_{C^{1}(\bar{D})} \leq N(K, d) .
$$

Near $\partial D$ let $\psi_{2}=\operatorname{dist}(x, \partial D)$ if $x \in \bar{D}, \psi_{2}=-\operatorname{dist}(x, \partial D)$ if $x \notin D$. By a standard argument in [15], we can define $\psi_{1}=\psi_{2}-t \psi_{2}^{2}$ near $\partial D$ with $t$ sufficiently large and continue $\psi_{1}$ in an appropriate manner such that

$$
\left\{x \in \mathbb{R}^{d}: \psi_{1}(x)>0\right\}=D,\left\|\psi_{1}\right\|_{C^{3,1}\left(\mathbb{R}^{d}\right)} \leq N(K, d)
$$

and we can find $\rho=\rho(K, d)>0$ such that for any $x \in \bar{\Delta}_{\rho},-\psi_{1, x x}$ is in $C_{m}$, where

$$
\Delta_{\rho}=\{x \in D: \operatorname{dist}(x, \partial D)<\rho\} .
$$

Denote $\Omega$ to be the closure of $\left\{K(v) / \operatorname{Tr}(K(v)) \mid v \in C_{m}\right\}$. Obviously, $\Omega$ is a compact set. Owing to Lemma 4.3, by a compactness argument we obtain the following corollary.

Corollary 4.8. There exist a $\delta=\delta(K, d, \rho)>0$, such that for any $v \in \Omega$ and $x \in \bar{\Delta}_{\rho}$ we have $\operatorname{Tr}\left(v \psi_{1, x x}(x)\right)<-\delta$. Especially, for any $w \in C_{m}$ and $x \in \bar{\Delta}_{\rho}$, we have $L^{w} \psi_{1}(x)<-\delta$. Moreover, for any $x \in \partial D,\left|\psi_{1 x}\right|=1$.

Proof. For any $x \in \bar{\Delta}_{\rho}$ we can find a number $\varepsilon>0$ such that $-\psi_{1, x x}-$ $\varepsilon I \in C_{m}$. Then we have

$$
\operatorname{Tr}\left(v\left(-\psi_{1, x x}-\varepsilon I\right)\right) \geq 0, \quad \operatorname{Tr}\left(v\left(-\psi_{1, x x}\right)\right)=\varepsilon>0 .
$$

Because both $\Omega$ and $\bar{\Delta}_{\rho}$ are compact, there exists $\delta>0$ such that $\operatorname{Tr}\left(v \psi_{1, x x}(x)\right)<-\delta$. The last assertion follows immediately from the definition of $\psi_{1}$.

Let $\psi_{0}=(2 R+1)^{2}-\left|x-x_{0}\right|^{2}$, where $R$ is the diameter of $D$ and $x_{0}$ is a point in $D$. Since $\operatorname{Tr}(a(w))=1$, it holds that $L^{w} \psi_{0}=-2$ for any $w \in C_{m}$. Thus Assumption 1.2 (a), (b) in [18] are satisfied and the following lemma is proved in [18, Lemma 1.1.

Lemma 4.9. There exist $\psi \in C^{3,1}$ and $\delta=\delta(K, d)>0$ satisfying (i) $\left\{x \in \mathbb{R}^{d}: \psi(x)>0\right\}=D$, (ii) $\left|\psi_{x}\right|>\delta$ on $\partial D$, (iii) $\|\psi\|_{C^{3,1}\left(\mathbb{R}^{d}\right)} \leq$ $N(K, d)$ and (iv) for any $w \in C_{m}$ and $x \in D$ we have $L^{w} \psi(x)<-\delta$. 


\section{Estimates of $u$ AND $u_{x}$}

In this section, we will give some estimates of $u$ and $u_{x}$.

Theorem 5.1. For any $x \in D$, we have

$$
|u(x)-\phi(x)| \leq N(K, d) \psi(x) .
$$

Proof. Denote $\tilde{u}=u-\phi$. Since $P_{m}$ is a homogeneous polynomial of degree $m$, we have

$$
\begin{gathered}
P_{m, u_{x^{i} x^{j}}}\left(u_{x x}(x)\right) u_{x^{i} x^{j}}(x)=m P_{m}\left(u_{x x}(x)\right)=m g^{m-1}(x), \\
\operatorname{Tr}\left(a\left(u_{x x}(x)\right) \tilde{u}_{x x}\right)=m g^{m-1}(x) / \operatorname{Tr}\left(K\left(u_{x x}(x)\right)\right)-\operatorname{Tr}\left(a\left(u_{x x}(x)\right) \phi_{x x}\right) .
\end{gathered}
$$

From the positiveness of $K\left(u_{x x}\right)$, equality (5.1) can be looked at as a second order elliptic equation of $\tilde{u}$. Due to (4.13), the right hand side of (5.1) is bounded by a constant depending only on $K, d$ and $m$. Lemma 4.9 implies

$$
\operatorname{Tr}\left(a\left(u_{x x}(x)\right) \psi_{x x}(x)\right)<-\delta .
$$

After using the comparison principle, we get what we expected.

Theorem 5.2. We have $\sup _{D}\left|u_{x}\right| \leq N$, where $N$ depends only on $K$ and $d$.

Proof. After differentiating (4.1) in the direction $\xi$, we get

$$
a^{i j}\left(u_{x x}(x)\right) u_{(\xi) x^{i} x^{j}}(x)=\frac{m-1}{d-m+1} g_{(\xi)}(x) g^{m-2}(x) P_{m-1}^{-1}\left(u_{x x}(x)\right) .
$$

Owing to (2.6) and (4.13), the absolute value of the right-hand side of (5.2) is less than

$$
N\left|g_{(\xi)}(x)\right| g^{m-2}(x) P_{m-1}^{-1}\left(u_{x x}(x)\right) \leq N g^{m-3 / 2} P_{m-1}^{-1}\left(u_{x x}(x)\right) \mid \leq N .
$$

Again by using Lemma 4.9 and the comparison principle, we get

$$
\sup _{D}\left|u_{(\xi)}\right| \leq N+\sup _{\partial D}\left|u_{(\xi)}\right| .
$$

Upon using Lemma 5.1. we get the estimate of the first derivative on the boundary:

$$
\left|u_{(\xi)}(x)\right| \leq N(K, d) \quad \forall x \text { on } \partial D,
$$

and the lemma is proved.

We also need a lower bound for the normal first order derivative $D_{n} u$ on the boundary $\partial D$. 
Lemma 5.3. Under Assumption [2.3, for any $x \in \partial D$ we have

$$
D_{n} u(x) \leq-\gamma<0,
$$

where $n$ is the inner normal of $\partial D$ at point $x$ and $\gamma$ is a constant depending only on $D, K$ and $d$.

Proof. From (4.7) with $w=I_{d}$ we get

$$
\Delta u(x) \geq N(m, d) g^{1-1 / m}(x) .
$$

Because $g$ is not always equal to 0 in $D$ and $u=0$ on $\partial D$, by the strong maximum principle, we get $u$ is strictly positive in $D$.

Since $D$ is a $C^{3,1}$ domain, we can find two positive numbers $\varepsilon_{1}$ and $\varepsilon_{2}$ depending only on $K$ satisfying: (i) for any $x \in \partial D$, there is a ball $B_{x}$ of radius $\varepsilon_{1}$ which is inside $D$ and $\partial B_{x}$ and $\partial D$ are tangent at $x$; (ii) for any $x \in \partial D$, if we look at $x$ as "north poll", then the south half ball of $B_{x}$ is in $D \backslash \Delta_{\varepsilon_{2}}$.

Next, we claim that there exists $\gamma_{0}=\gamma_{0}(D, d, K)>0$ such that

$$
u(x) \leq-\gamma_{0} \quad \text { in } D \backslash \Delta_{\varepsilon_{2}} .
$$

We prove this by contradiction. If this is not true, we can find two sequences $x_{j} \in \bar{D}, g_{j} \in C^{2}(\bar{D})$ such that

$$
\left\|g_{j}\right\|_{C^{1}(\bar{D})} \leq K, \quad g_{j}\left(x_{j}\right) \geq 1 / K
$$

and also $u_{j}$ solves (4.1)-(4.2) with $g_{j}$ in place of $g$ such that

$$
\sup _{D \backslash \Delta_{\varepsilon_{2}}} u_{j} \rightarrow 0, \quad \text { as } j \rightarrow+\infty \text {. }
$$

Because $\bar{D}$ is compact, after passing to a subsequence, we may assume $x_{j}$ converges to a point $x_{0} \in \bar{D}$. Due to (5.4) for a small neighborhood $U_{x_{0}}$ of $x_{0}$ we have $\inf _{U_{x_{0}} \cap \bar{D}} g_{j} \geq 1 /(2 K)$ for all $j$ large enough. Let $u_{0}$ be the solution of

$$
\Delta u_{0}(x)=N(m, d)\left(I_{U_{x_{0}} \cap D} /(2 K)\right)^{1-1 / m}(x)
$$

with zero boundary data, where $N(m, d)$ is the same positive constant as that of (5.3). Then by the comparison principle, we get

$$
u_{j}(y) \leq u_{0}(y) \quad \text { in } D \text {. }
$$

However, by the strong maximum principle, $u_{0}$ is strictly negative in $D$ and bounded away from 0 on $D \backslash \Delta_{\varepsilon_{2}}$, which contradicts (5.5).

Now for any $x \in \partial D$, we consider $u$ in the ball $B_{x}$. By the previous proof, we have $u \leq-\gamma_{0}$ on the south half sphere and $u(x)=0$. Moreover, $u$ is subharmonic in $B_{x}$. Denote $v$ to a harmonic function in $B_{x}$ with boundary data 0 on the north half sphere and $-\gamma_{0}$ on the south half sphere. By the comparison principle again we have $u(y) \leq v(y)$ in 
$B_{x}$, and therefore $D_{n} u(x) \leq D_{n} v(x)<0$. Actually, by our construction of $v, D_{n} v(x)$ only depends on $K$ and $d$ (not $x$ ). This completes the proof of the lemma.

\section{INTERIOR SECOND ORDER DERIVATIVES}

Here, our goal is to firstly give an interior estimate of the second order derivatives of the solution via the estimates on the boundary of the second order derivatives.

Note that for any function $H(\alpha, x)$ which is twice differentiable in $x$, if $\inf _{\alpha \in A} H(\alpha, x)$ is also twice differentiable, then for any $\xi \in \mathbb{R}^{d}$ pointwisely we have

$$
\left(\inf _{\alpha \in A} H(\alpha, x)\right)_{(\xi)(\xi)} \leq H_{(\xi)(\xi)}\left(\alpha_{0}, x\right), \quad \Delta\left(\inf _{\alpha \in A} H(\alpha, x)\right) \leq \Delta H\left(\alpha_{0}, x\right),
$$

where $\alpha_{0} \in A$ such that $H\left(\alpha_{0}, x\right)=\inf _{\alpha \in A} H(\alpha, x)$. After differentiating (4.8) twice in the direction $\xi$, we get

$$
\begin{gathered}
\left(P_{m}^{1-1 / m} P_{m-1}^{-1}\right)\left(u_{x x}(x)\right) \frac{m-1}{d-m+1}\left[g_{(\xi)(\xi)} g^{-1 / m}(x)\right. \\
\left.-(1 / m) g_{(\xi)}^{2} g^{-1-1 / m}(x)\right] \leq a^{i j}\left(u_{x x}(x)\right) u_{x^{i} x^{j}(\xi)(\xi)}(x) .
\end{gathered}
$$

Because of (2.6), (4.1) and (4.6), we obtain

$$
P_{m, u_{x^{i} x^{j}}}\left(u_{x x}(x)\right) u_{x^{i} x^{j}(\xi)(\xi)}(x) \geq-N g^{m-2}(x) .
$$

Observe that by Lemma 4.1, (6.1) is an elliptic equation in D. Combining (4.12) with the comparison principle for the elliptic equations in $D^{\prime}$, we get an upper estimate

$$
u_{(\xi)(\xi)}(x) \leq N+\sup _{\partial D^{\prime}} u_{(\xi)(\xi)}(x) \leq N+\sup _{\partial D} u_{(\xi)(\xi)}(x) .
$$

The last inequality is because $\left|u_{(\xi)(\xi)}(x)\right| \leq d-1$ on $\partial D^{\prime} \cap D$.

To obtain the lower estimate, it remains to use (4.10) again. As a conclusion, we get

Theorem 6.1. Let $u \in C^{4}(D) \cap C^{2}(\bar{D})$ be the solution of $\sqrt{4.1}$ ) in $D$ and satisfies $\left(u_{x x}(x)\right) \in C_{m}$ in $D$. Then

$$
\sup _{D}\left|D^{2} u\right| \leq N\left(1+\sup _{\partial D}\left|D^{2} u\right|\right)
$$

where $N$ is a constant depending only on $K$ and $d$.

Remark 6.2. It turns out that to get (6.3) it suffices to assume $\Delta g$ to be bounded from below. Indeed, under this condition, instead of (6.1) and (6.2) we have

$$
P_{m, u_{x^{i} x^{j}}}\left(u_{x x}(x)\right) \Delta u_{x^{i} x^{j}}(x) \geq-N g^{m-2}(x),
$$




$$
\Delta u(x) \leq N+\sup _{\partial D^{\prime}} \Delta u(x) \leq N+\sup _{\partial D} \Delta u(x) .
$$

The last estimate together with (4.11) yields (6.3).

\section{BOUNDARY SECOND ORDER DERIVATIVES}

We remark here that for the problem with homogeneous boundary condition the estimate of the second order derivatives on the boundary follows from the arguments in Section 5 of $[3]^{1}$ by applying Lemma 5.3 instead of the usual Hopf lemma. Here we give some details for the sake of completeness. While estimating the mixed second derivates, we use Krylov's approach in [16] and [17.

For any $x \in \partial D$, after a shift of the origin and an orthogonal transformation, we may suppose $x$ is the origin and $x^{n}$-axis is the inner normal. By further transforming the coordinate $x^{\prime}=\left(x^{1}, \ldots, x^{n-1}\right)$, we can assume in a small neighborhood $U_{0}$ of $x, \partial D$ can be represented by $x^{n}=\bar{\psi}\left(x^{\prime}\right)$ and $u_{x_{i} x_{j}}(0)=0$ for $i \neq j, i, j=1, \ldots, n-1$. Here $x^{n}-\bar{\psi}\left(x^{\prime}\right)$ is in the class of $C^{3,1}\left(\bar{U}_{0}\right)$ and

$$
\left\|x^{n}-\bar{\psi}\left(x^{\prime}\right)\right\|_{C^{3,1}\left(\bar{U}_{0}\right)} \leq N(d, K), \quad \nabla \bar{\psi}(0)=0 .
$$

Then it suffices to estimate $u_{x^{j} x^{j}}(0), u_{x^{j} x^{n}}(0)$ and $u_{x^{n} x^{n}}(0)$, where $j=$ $1, \ldots, n-1$.

The estimation of the tangential second order derivatives on the boundary is standard (cf. [3], 7] or [17]). We differentiate the equality

$$
u\left(x^{\prime}, \bar{\psi}\left(x^{\prime}\right)\right)=0
$$

twice with respect to $x^{j}, j=1, \ldots, n-1$, and get

$$
u_{x^{n}}(0) \bar{\psi}_{x^{j} x^{j}}(0)+u_{x^{j} x^{j}}(0)=0,
$$

which along with Theorem 5.2 gives a bound for $u_{x^{j} x^{j}}(0)$.

Next, let's estimate the mixed derivatives $u_{x^{j} x^{n}}(0)$. We start with introduce a few more objects. Denote $\mathbb{A}$ to be the space of all skewsymmetric matrices and for $p \in \mathbb{A}$ we set

$$
a(w, p)=a\left(e^{p} w e^{-p}\right)=e^{p} a(w) e^{-p}, \sigma=\sqrt{2 a}, f(w, p, x)=f(w, x) .
$$

For $\xi \in \mathbb{R}^{d}$, we also define $P(w, x) \xi=P(x) \xi$ with value in $\mathbb{A}$ by the formula

$$
[P \xi]_{i j}=\psi_{1 x^{i}(\xi)} \psi_{1 x^{j}}-\psi_{1 x^{i}} \psi_{1 x^{j}(\xi)} .
$$

Since $e^{p} C_{m} e^{-p}=C_{m}$ and $f\left(e^{p} w e^{-p}, x\right)=f(w, x)$, we have

$$
0=\inf _{w \in C_{m}}\left\{a^{i j}(w) u_{x^{i} x^{j}}(x)+f(w, x)\right\}
$$

\footnotetext{
${ }^{1}$ We are grateful to the referee for pointing this out.
} 


$$
=\inf _{w \in C_{m}, p \in \mathbb{A}}\left\{a^{i j}(w, p) u_{x^{i} x^{j}}(x)+f(w, p, x)\right\} .
$$

Owing to the proof of Theorem 5.9 of [15], there exist positive numbers $\delta_{1}$ and $\delta_{2}$ depending only on $K$ and $d$ such that by taking $B_{1}:=\delta_{1} I_{d}$ the following assumption is satisfied. This assumption is exactly Assumption $1.2(\mathrm{~d})$ of [17] with $K$ there equal to 0.

Assumption 7.1. For any $x \in \partial D, \xi \perp \psi_{1 x}(x),|\xi|=1, w \in C_{m}, p=0$ we have $\left(B_{1} \xi, \xi\right)=\delta_{1}$ and

$$
\begin{gathered}
L^{w} \psi_{1}+a^{i j} B_{1 i j} \leq-\delta_{2} \\
\left(B_{1} \xi, \xi\right) L^{w} \psi+\sum_{k}\left(\partial(\xi) \psi_{1\left(\sigma^{k}\right)}\right)^{2}+2\left(B_{1} \xi, \sigma^{k}\right) \partial(\xi) \psi_{1\left(\sigma^{k}\right)} \leq-\delta_{2},
\end{gathered}
$$

where

$$
\partial(\xi) \psi_{1\left(\sigma^{k}\right)}=\left.\frac{d}{d h} \psi_{1 x^{i}}(x+h \xi) \sigma^{i k}(w, h P(w, x) \xi)\right|_{h=0}
$$

The estimation of the mixed second order derivatives is a direct application of Theorem 1.10 of [17]. First notice that, as we mentioned before, (4.8) is equivalent to (4.9), which is uniformly elliptic. Next, to estimate the mixed second order derivatives we consider the function $\bar{u}=u / \psi$, which satisfies a higher dimensional elliptic Bellman equation on an auxiliary manifold. Then the problem is reduced to the estimation of tangential first order derivatives of $\bar{u}$ on the manifold. In turn, actually it suffices to have $f(w, \cdot)$ to be in $C^{1}(\bar{D})$, which is already satisfied in our case due to (4.15). As a conclusion we get

Lemma 7.2. Under our assumptions, there exist positive constants $\rho=\rho(K, d)$ and $N=N(K, d)$ such that for any $x \in \partial D$ and unit $\tau \perp \psi_{x}(x)$ we have

$$
\left|u_{(\tau)(n)}(x)\right| \leq N\left(1+\max _{\partial D(\rho)}\left(|u|+\left|u_{x}\right|\right)\right] .
$$

This immediately implies the estimate of $u_{x^{j} x^{n}}(0), j=1, \ldots, n-1$.

We use the equation (2.4) itself to estimate the normal second order derivative $u_{x^{n} x^{n}}(0)$. Equation (2.4) at the origin can be rewritten as

$$
u_{x^{n} x^{n}}(0) P_{m-1, d-1}\left(u_{x^{1} x^{1}}(0), \cdots, u_{x^{n-1} x^{n-1}}(0)\right)+G=g^{m-1}(0),
$$

where $G$ is a sum of products of $u_{x^{j} x^{j}}(0)$ and $u_{x^{j} x^{n}}(0), j=1, \cdots, n-1$. By the results of Section [7] we have

$$
|G| \leq N(K, d) \text {. }
$$

Due to (2.1), (17.1) and Lemma 5.3. we get

$$
P_{m-1, d-1}\left(u_{x^{1} x^{1}}(0), \cdots, u_{x^{n-1} x^{n-1}}(0)\right)
$$




$$
\begin{aligned}
& =\left(-u_{x^{n}}(0)\right)^{m-1} P_{m-1, d-1}\left(\bar{\psi}_{x^{1} x^{1}}(0), \cdots, \bar{\psi}_{x^{n-1} x^{n-1}}(0)\right) \\
\geq & \delta(D, K, d) P_{m-1, d-1}\left(\kappa^{1}, \cdots, \kappa^{d-1}\right) \geq \delta(D, K, d)>0 .
\end{aligned}
$$

Combining (7.2), (7.3) and (17.4) together yields

$$
\left|u_{x^{n} x^{n}}(0)\right| \leq N(D, K, d) .
$$

Thus for the non-degenerate case $g>0$ in $D$, we get the estimate for $\left|u_{x x}\right|$ on $\partial D$, and subsequently in $D$ by Theorem 6.1. For the general case, we only have to use Lemma 4.6 .

Remark 7.3. It is worth noting that when we estimate the second order derivatives on the boundary $\partial D$, we only use the fact

$$
\|f(w, \cdot)\|_{C^{1}(\bar{D})} \leq N(K, d) .
$$

So to get the boundary estimates, it suffices to assume $g^{2(m-1) / m} \in$ $C^{1}(\bar{D})$, which in general is weaker than the condition that $g$ itself is in $C^{1}(\bar{D})$.

Remark 7.4. Our method can be carried over to a larger class of Hessian equations

$$
P_{m}\left(u_{x x}+h\right)=g^{m-1}
$$

with zero boundary condition, where $h \in C^{1,1}\left(\bar{D}, \bar{C}_{m}\right)$ is a $\bar{C}_{m}$-valued function satisfying the following condition:

$$
\begin{aligned}
& \operatorname{Tr} h \leq \frac{m d}{d-m+1} \frac{P_{m}^{1-1 / m}(I)}{P_{m-1}(I)} g^{1-1 / m}, \\
& \operatorname{Tr} h \not \equiv \frac{m d}{d-m+1} \frac{P_{m}^{1-1 / m}(I)}{P_{m-1}(I)} g^{1-1 / m} .
\end{aligned}
$$

Naturally, we look for solutions such that $u_{x x}+h \in \bar{C}_{m}$ (a.e.).

Indeed, as we mentioned before, for large $t$ on $\partial D$ we have $-\psi_{1, x x} \in$ $C_{m}$. Due to Corollary 4.2 on $\partial D$ we have $\operatorname{diag}\left\{\kappa^{1}, \cdots, \kappa^{d-1}\right\} \in C_{m-1}$. As before, we can rewrite (7.6) as a Bellman equation

$$
\inf _{w \in C_{m}}\left\{L^{w} u(x)+f(w, x)+\operatorname{Tr}(a(w) h(x))\right\}=0,
$$

where $L^{w}$ and $f(w, x)$ are defined in the same way as in (4.8). By the same method we can get the estimates of $u$ and $u_{x}$, and reduce the interior estimate of $u_{x x}$ to the estimates of $u_{x x}$ on the boundary.

Under the linear transformation introduced at the beginning of Section [7. $h(0)$ becomes $\bar{h}(0)$. Denote $\bar{h}_{n}$ to be the $(d-1) \times(d-1)$ submatrix obtained by deleting the $n$th row and $n$th column of $\bar{h}(0)$. By Corollary $4.2, \bar{h}_{n}$ is in $\bar{C}_{m-1, d-1}$. After estimating the tangential and mixed second order derivatives on the boundary in a similar way, 
we can obtain the estimate of $u_{x^{n} x^{n}}(0)$ by using the equation (17.6) itself and the inequality

$$
\begin{gathered}
P_{m-1, d-1}\left(\operatorname{diag}\left\{u_{x^{1} x^{1}}(0), \cdots, u_{x^{n-1} x^{n-1}}(0)\right\}+\bar{h}_{n}\right) \\
\geq P_{m-1, d-1}\left(\operatorname{diag}\left\{u_{x^{1} x^{1}}(0), \cdots, u_{x^{n-1} x^{n-1}}(0)\right\}\right) \\
=P_{m-1, d-1}\left(\operatorname{diag}\left\{-u_{x^{n}}(0) \bar{\psi}_{x^{1} x^{1}}(0), \cdots,-u_{x^{n}}(0) \bar{\psi}_{x^{n-1} x^{n-1}}(0)\right\}\right) \\
\geq\left(-u_{x^{n}}(0)\right)^{m-1} P_{m-1, d-1}\left(\bar{\psi}_{x^{1} x^{1}}(0), \cdots, \bar{\psi}_{x^{n-1} x^{n-1}}(0)\right) \geq \delta(D, K, d)>0 .
\end{gathered}
$$

Here in the first step we use the inequality

$$
P_{m-1}(A+B) \geq P_{m-1}(A)
$$

for any $A, B \in \bar{C}_{m-1}$ (cf. Lemma 4.3 or (4.4)).

\section{Proof of Lemma 4.6}

Let $g_{n}$ be a sequence of strictly positive functions in $C^{2}(\bar{D})$ such that for $n=1,2, \cdots$, the functions $g_{n}(x)+2 K|x|^{2}$ are convex on $\bar{D}$ and

$$
\inf _{D} g_{n} \geq 1 /(2 n), \quad\left\|g_{n}\right\|_{C^{1}(\bar{D})} \leq 2 K, \quad\left\|g_{n}-g\right\|_{C^{0}(\bar{D})} \leq 1 / n .
$$

By our assumption there exists $N=N(D, d, K)$ such that

$$
\left\|v_{n}\right\|_{C^{2}(\bar{\Omega})} \leq N(D, d, K), \quad m=1,2, \cdots,
$$

where $v_{n} \in C^{2}(\bar{D}) \cap C^{4}(D)$ is the solution of (4.1) with $g_{n}$ in place of $g$ and with zero Dirichlet boundary condition. By Arzelà-Ascoli theorem, after passing to a subsequence if necessary, $\left\{v_{n}\right\}$ converges in $C^{1}(\bar{D})$ to a function $v$. Again by (8.1) we get $v \in C^{1,1}(\bar{D})$ and

$$
\|v\|_{C^{1,1}(\bar{\Omega})} \leq N(D, d, K) .
$$

Owing to the uniqueness of the admissible weak solution, we obtain

$$
u=v, \quad\|u\|_{C^{1,1}(\bar{\Omega})} \leq N(D, d, K) .
$$

This completes the proof of (i).

To prove (ii) we use the idea in the proof of Lemma 7.3.4 14. Let $\Omega$ be a countable dense subset of $C_{m}$. Obviously, one has

$$
\inf _{w \in C_{m}}\left[L^{w} u+f\right]=\inf _{w \in \Omega}\left[L^{w} u+f\right] .
$$

For any $w \in \Omega$, in $D$ it holds that

$$
L^{w} v_{n}(x)+f_{n}(w, x) \geq 0,
$$

where

$$
f_{n}(w, x)=-m(d-m+1)^{-1} P_{m}^{1-1 / m}(w) P_{m-1}^{-1}(w) g_{n}^{1-1 / m}(x) .
$$


After multiplying (8.2) by a nonnegative function $\eta \in C_{0}^{\infty}(D)$, integrating by parts, passing to the limit over the sequence $n$ and integrating by parts again, we obtain

$$
\int_{D}\left[a^{i j}(w) \eta(x) u_{x^{i} x^{j}}(x)+\eta(x) f(w, x)\right] d x \geq 0 .
$$

Because $\eta \in C_{0}^{\infty}(D)$ is arbitrary, we further get

$$
a^{i j}(w) u_{x^{i} x^{j}}(\cdot)+f(w, \cdot) \geq 0 \text { a.e. in } D .
$$

Since $\Omega$ is a countable set, we reach

$$
\sup _{w \in \Omega}[L u(x)+f(w, x)] \geq 0 \quad \text { a.e. in } D .
$$

Next we prove the opposite inequality. Here we use again the method by which Lemma 7.3.4 of [14] is proved. Let $\varepsilon<1$ be a positive number. Recall that $v_{n}$ satisfies the Bellman equation

$$
\inf _{w \in C_{m}}\left\{L^{w} v_{n}(x)+f_{n}(w, x)\right\}=0 .
$$

Given any $n$ such that $1 / n<\varepsilon$, we have

$$
\begin{gathered}
\inf _{w \in C_{m}}\left\{L^{w} v_{n}(x)+\varepsilon \Delta v_{n}(x)+f(w, x)\right\} \\
\leq \sup _{w \in C_{m}}\left\{\varepsilon \Delta v_{n}(x)+f(w, x)-f_{n}(w, x)\right\} \leq N(D, d, K) \varepsilon, \\
\inf _{w \in C_{m}}\left\{L^{w} v_{n}(x)+\varepsilon \Delta v_{n}(x)+f(w, x)-N(D, d, K) \varepsilon\right\} \leq 0 .
\end{gathered}
$$

Note that the elliptic operator $\inf _{w \in C_{m}}\left[L^{w}+\varepsilon \Delta\right]$ is uniformly nondegenerate. Owning to Theorem 3.6.3 [14], after passing to the limit over the sequence $n$ we obtain

$$
\inf _{w \in C_{m}}\left\{L^{w} u(x)+\varepsilon \Delta u(x)+f(w, x)-N(D, d, K) \varepsilon\right\} \leq 0 \text { a.e. in } D .
$$

Letting $\varepsilon \downarrow 0$ yields an inequality opposite to (8.3). The lemma is proved.

\section{REFERENCES}

[1] L.A. Caffarelli, L. Nirenberg, J. Spruck, The Dirichlet problem for nonlinear second-order elliptic equations, I: Monge-Ampère equation, Comm. Pure Appl. Math., 37 (1984), 369-402.

[2] L.A. Caffarelli, J.J. Kohn, L. Nirenberg, J. Spruck, The Dirichlet problem for nonlinear second-order elliptic equations, II: Complex Monge-Ampère equation and uniformly elliptic equations, Comm. Pure Appl. Math., 38 (1985), 209-252.

[3] L.A. Caffarelli, L. Nirenberg, J. Spruck, The Dirichlet problem for nonlinear second-order elliptic equations, III. Functions of the eigenvalues of the Hessian, Acta Math., 155 (1985), 261-301.

[4] L.A. Caffarelli, L. Nirenberg, J. Spruck, The Dirichlet problem for degenerate Monge-Ampère equations. Rev. Mat. Iberoamericana, 2 (1986), 19-27. 
[5] L. Garding, An inequality for hyperbolic polynomials, J. Math. Mech., 8 (1959), 957-965.

[6] G. Gilbarg, N.S. Trudinger, Elliptic Partial Differential Equations of Second Order, 2nd edition, Springer-Verlag, Berlin-New York, 1983.

[7] P. Guan, N.S. Trudinger, X. Wang, On the Dirichlet problem for degenerate Monge-Ampère equations, Acta Math., 182 (1999), no.1, 87-104.

[8] L. Hömander, The analysis of linear partial differential operators, Vols. 1,2, Springer, New York, 1990.

[9] J. Hong, The estimates near the boundary for solutions of Monge-Ampre equations. J. Partial Differential Equations, 7 (1994), 97-110.

[10] N.M. Ivochkina, A priori estimate of $\|u\|_{C^{2}(\bar{\Omega})}$ of convex solutions of the Dirichlet problem for the Monge-Ampère equation, Zap. Nauchn. Sem. Leningrad. otdel. Mat. Inst. Steklov. 96 (1980), 69-79 (Russian); English transl. J. Soviet Math., 21 (1983), 689-697.

[11] N. Ivochkina, N.S. Trudinger, X. Wang, The dirichlet problem for degenerate Hessian equations, Comm. Partial Differential Equations, 29 (2004), No. 1-2, 219-235.

[12] N.V. Krylov, Controlled diffusion processes, Spring-Verlag, New York, 1980.

[13] N.V. Krylov, Boundedly inhomogeneous elliptic and parabolic equations in a domain, Izvv. Akad. Nauk SSSR, 47 (1983), 75-108 (Russian); English transl. Math. USSR-Izv., 22 (1984), 67-98.

[14] N.V. Krylov, Nonlinear elliptic and parabolic equations of second order, Nauka, Moscow, 1985; English transl., Reidel, Dordrecht, 1987.

[15] N.V. Krylov, On the general notion of fully nonlinear second order elliptic equation, Trans. Amer. Math. Soc., 347 No. 3, (1995), 857-895.

[16] N.V. Krylov, Weak interior second order derivative estimates for degenerate nonlinear elliptic equations, Differential and Integral Equations, 7 (1994), 133156.

[17] N.V. Krylov, A theorem on degenerate elliptic bellman equations in bounded domains, Differential and Integral Equations, 8 (1995), 961-980.

[18] N.V. Krylov, Smoothness of the payoff function for a controllable process in a domain, Izv. Akad. Nauk SSSR, ser. math., 53 (1989), 66-96, in Russian; English translation: Math.USSR-Izv., 34, (1990), 65-95.

[19] Pogorelov, A., The Minkowski multidimensional problems, J. Wiley, New York, 1978.

[20] M.V. Safonov, The classical solution of Bellman's elliptic equation, Dokl. Akad. Nauk SSSR, 278, No. 4 (1984), 810-813; English transl., Soviet Math. Dokl., 30 (1984), No. 42, 482-485.

[21] N.S. Trudinger, On the Dirichlet problems for Hessian equations, Acta Math., 175 (1995), 151-164.

[22] N.S. Trudinger, Weak solutions of Hessian equations, Comm. Partial Differential Equations, 22 (1997), no. 7-8, 1251-1261.

[23] X. Wang, Some counterexamples to the regularity of Monge-Ampre equations, Proc. Amer. Math. Soc., 123 (1995), 841-845

(H. Dong) 127 Vincent Hall, University of Minnesota, Minneapolis, MN 55455, USA

E-mail address: hjdong@math.umn.edu 